\title{
Cytoreductive nephrectomy for metastatic renal cell carcinoma, the ultimate urologic 'Choosing Wisely' campaign: a narrative review
}

\author{
Alexandra L. Tabakin ${ }^{1}$, Mark N. Stein ${ }^{2}$, Christopher B. Anderson ${ }^{3}$, Charles G. Drake ${ }^{2}$ Eric A. Singer ${ }^{1}$ \\ ${ }^{1}$ Section of Urologic Oncology, Rutgers Cancer Institute of New Jersey and Rutgers Robert Wood Johnson Medical School, New Brunswick, NJ \\ 08903, USA; ${ }^{2}$ Division of Medical Oncology, Columbia University College of Physicians and Surgeons, New York, NY 10032, USA; ${ }^{3}$ Department of \\ Urology, Columbia University College of Physicians and Surgeons, New York, NY 10032, USA \\ Contributions: (I) Conception and design: AL Tabakin, EA Singer; (II) Administrative support: MN Stein, CB Anderson, CG Drake, EA Singer; \\ (III) Provision of study materials or patients: None; (IV) Collection and assembly of data: AL Tabakin, MN Stein, EA Singer; (V) Data analysis and \\ interpretation: AL Tabakin, MN Stein, EA Singer; (VI) Manuscript writing: All authors; (VII) Final approval of manuscript: All authors. \\ Correspondence to: Eric A. Singer, MD, MA, MS, FACS. Associate Chief of Urology and Urologic Oncology, Associate Professor of Surgery and \\ Radiology, Rutgers Cancer Institute of New Jersey, 195 Little Albany Street, New Brunswick, NJ 08903, USA. Email: singerea@cinj.rutgers.edu.
}

\begin{abstract}
In the early 2000s, cytoreductive nephrectomy in addition to systemic cytokines became standard of care for treating metastatic renal cell carcinoma. Since that time, the development of novel systemic targeted therapies and immuno-oncologic agents have challenged the utility of cytoreductive nephrectomy in clinical practice. In 2019, the controversial CARMENA study was published, providing the first level one evidence suggesting that cytoreductive nephrectomy combined with targeted therapy yielded no survival advantage over targeted therapy alone in intermediate and poor risk metastatic renal cell carcinoma patients. Later that year, the SURTIME trial demonstrated that patients undergoing targeted therapy with delayed nephrectomy maintained a survival advantage over those that underwent upfront cytoreductive nephrectomy followed by targeted therapy. Both of these studies underscored the importance of patient selection and timing of cytoreductive nephrectomy and systemic therapy. As new immuno-oncologic agents are trialed, particularly in combination, the role of cytoreductive nephrectomy will continue to be questioned. In this narrative review, we discuss the evolution of the role of cytoreductive nephrectomy in treating metastatic renal cell carcinoma through the context of the ever-changing landscape of targeted therapies and immunooncologic agents. We assess the evidence for cytoreductive nephrectomy with respect to patient factors, timing of surgery, and combination with other therapies.
\end{abstract}

Keywords: Metastatic renal cell carcinoma (mRCC); cytoreductive nephrectomy; immunotherapy; immunooncology

Submitted Jun 16, 2020. Accepted for publication Oct 21, 2020.

doi: $10.21037 /$ tcr-20-2343

View this article at: http://dx.doi.org/10.21037/tcr-20-2343

\section{Introduction}

Renal cell carcinoma is the $8^{\text {th }}$ most common cancer in the United States with an overall 5-year survival rate of $75 \%(1,2)$. In the United States, $16 \%$ of patients initially present with metastatic renal cell carcinoma (mRCC) (3), and of these patients, approximately $12 \%$ survive five years (2).

Cytoreductive nephrectomy $(\mathrm{CN})$ is not offered as a curative surgery, but it can palliate hematuria, symptoms from neoplastic syndromes, or pain (4), and has been shown to prolong survival in the setting of systemic cytokines (5). Although $\mathrm{CN}$ has been reported to result in rare complete responses (6), it is frequently performed as an adjunct to systemic targeted therapy (TT) or immuno-oncology (IO) agents. The proposed mechanism of action of $\mathrm{CN}$ rests on 
the principle that RCC produces cytokines that interfere with host defenses. By removing a large percentage of the tumor burden, anti-neoplastic immune function is restored (7).

In this narrative review, we discuss how the role of $\mathrm{CN}$ has evolved alongside the development of TT, IO, and TT/IO combinations. We assess how to best utilize $\mathrm{CN}$ in contemporary practice with respect to timing of surgery and the use of systemic therapy, highlighting current clinical trials. We present the following article in accordance with the Narrative Review reporting checklist (available at http:// dx.doi.org/10.21037/tcr-20-2343).

\section{Methods}

Using PubMed, Google Scholar, and Wiley Online Library, we performed a non-systematic review of articles between January 1993 and September 2020. Search terms included combinations of the following terms: "metastatic renal cell carcinoma", "cytoreductive nephrectomy", "systemic therapy", "targeted therapy", and "immunotherapy". Articles selected were required to be original articles published in English. News articles, editorials, and unpublished works were excluded.

Information on clinical trials was collected from www. clinicaltrials.gov, which was accessed in April 2020. Trials were selected by using combinations of the search terms "renal cell carcinoma", "systemic therapy", and "cytoreductive nephrectomy". Trials were classified as completed (Table 1) if their status was listed as "completed" on www.clinicaltrials.gov. Trials were classified as ongoing (Table 2) if their status was listed as "active", "recruiting", "active, not yet recruiting", "active, not recruiting", or "suspended". Trials were excluded if they were listed as "terminated" or "withdrawn".

\section{Current guidelines for $\mathrm{CN}$}

According to the National Comprehensive Cancer Network (NCCN), select patients with resectable mRCC should be offered CN (19). Candidates should have good performance status (ECOG <2), good prognostic features, and no brain metastases. Patients experiencing symptoms caused by their renal cancer, such as hematuria, may also be offered $\mathrm{CN}$.

In light of results from the SURTIME and CARMENA trials, the European Association of Urology (EAU) updated their guidelines in 2018 for $\mathrm{CN}(16,17,20)$. The EAU now recommends performing immediate $\mathrm{CN}$ in patients with good performance status not requiring systemic therapy. For intermediate-risk patients with asymptomatic synchronous primary tumors, sunitinib should be offered as initial treatment, whereas $\mathrm{CN}$ as initial treatment is contraindicated; delayed $\mathrm{CN}$ can be offered in patients with a limited metastatic burden. Poor risk patients should never be offered $\mathrm{CN}$, as supported by both retrospective and prospective studies (20).

\section{Surgical considerations for $\mathbf{C N}$}

$\mathrm{CN}$ can be a complex operation associated with significant morbidity and mortality $(21,22)$. In a retrospective multiinstitutional analysis of 736 patients with mRCC undergoing $\mathrm{CN}, 10.9 \%$ of patients experienced intraoperative complications, the most common of which included bleeding (36\%), splenic laceration (19\%), and vascular injury $(16 \%)$. Predictors of intraoperative complications included performing a thrombectomy or adjacent organ removal. 217 patients (29.5\%) experienced postoperative complications, $6.1 \%$ of which were considered high grade (Clavien Dindo $\geq 3$ ). Most common complications were vascular (30\%), infectious (19\%), and cardiopulmonary (17\%). Estimated blood loss (OR 2.93; 95\% CI: 1.20-7.15; $\mathrm{P}=0.02)$ and surgeon $\mathrm{CN}$ case volume (OR $0.13 ; 95 \% \mathrm{CI}$ : $0.03-0.59 ; \mathrm{P}=0.009)$ were identified as significant predictors of high grade complications (22).

Another study retrospectively evaluating 294 patients who underwent CN between 1990 and 2009 demonstrated a $12 \%$ early overall and 5\% early major (Clavien Dindo $\geq 3$ ) complication rate. The most common complications included wound infection (3\%), acute renal failure (3\%), pulmonary embolism (2\%), and deep venous thrombosis $(2 \%)$. Three deaths $(1 \%)$ were reported in the immediate 30-day postoperative period. While most patients did not experience complications, $61 \%$ of patients pre-determined to be candidates for systemic therapy did not receive it within 60 days of surgery (21).

Traditionally performed via an open approach, data on outcomes associated with minimally-invasive $\mathrm{CN}$ are sparse. Primary renal tumors in patients with mRCC may have adverse features such as IVC thrombi, significant bulky adenopathy, or invasion into local structures, making surgery technically challenging. A 2016 multi-institutional study reported on 120 patients across three high volume centers who underwent either laparoscopic $(96.6 \%)$ or robotic (3.4\%) CN between 2001 and 2013. 28 patients $(23.3 \%)$ had postoperative classifications, nearly $30 \%$ of 


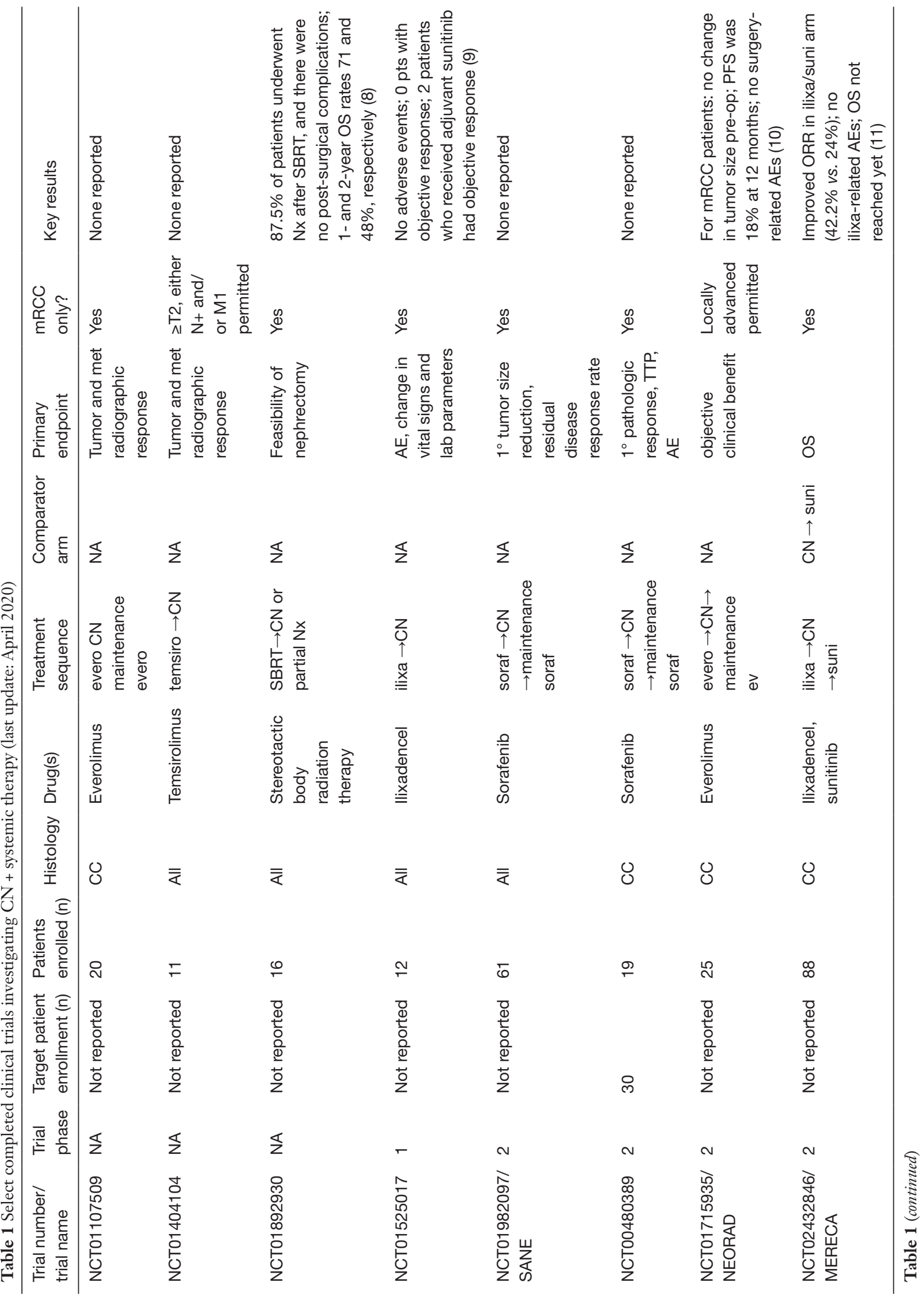




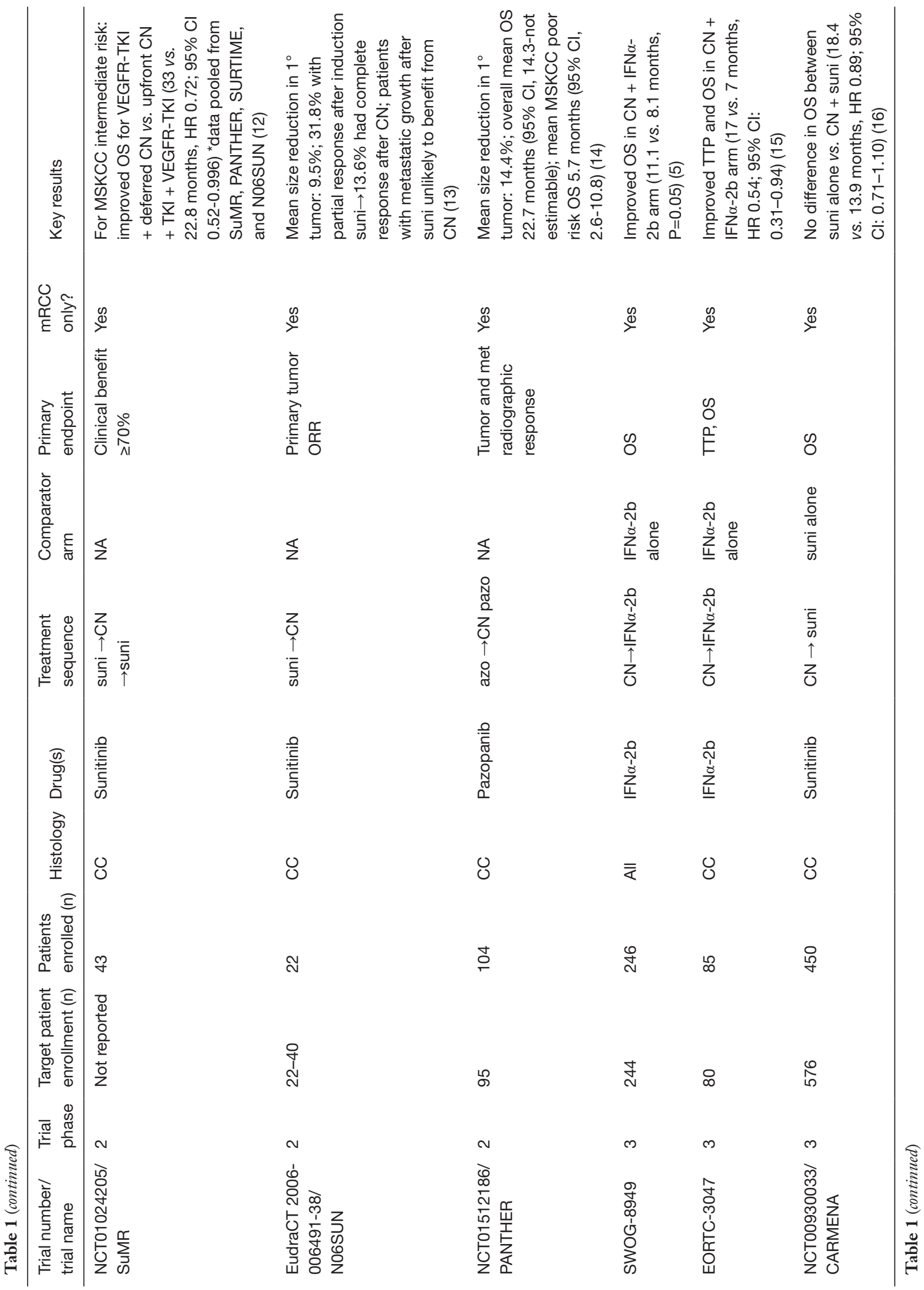




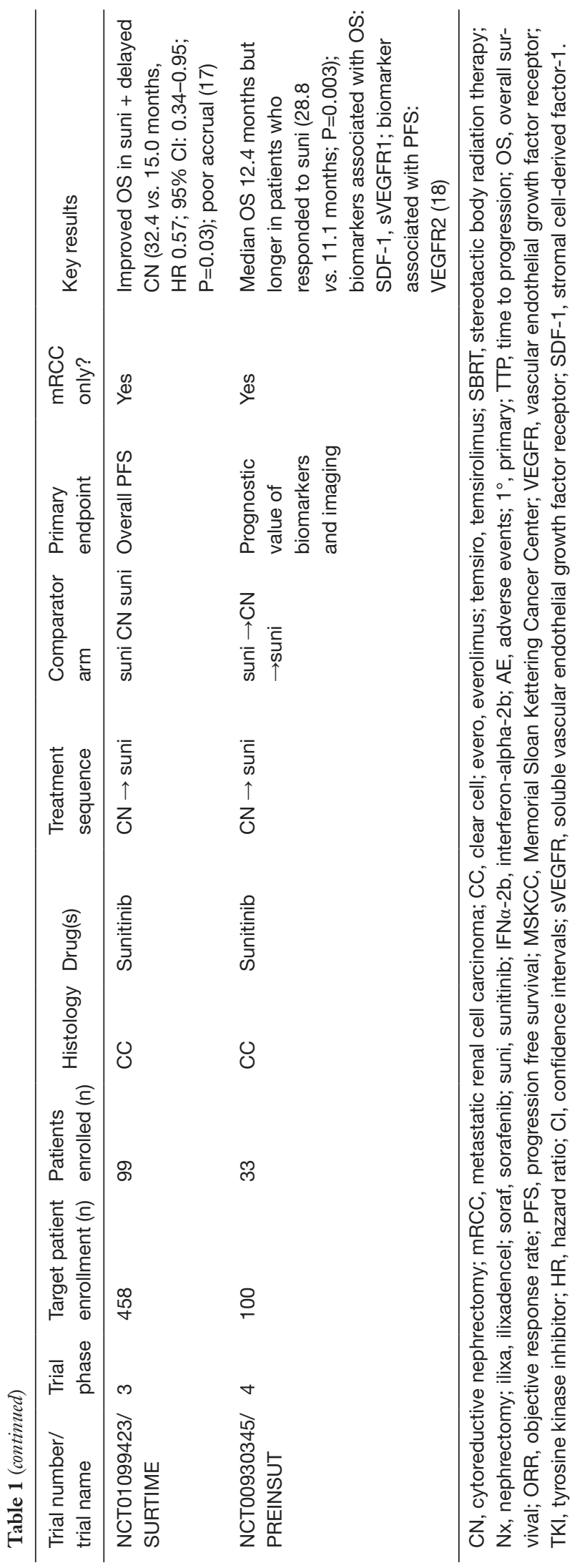

which were considered major (Clavien Dindo $\geq 3$ ). 4 patients $(3.3 \%)$ required conversion to open surgery (23). At this time, no randomized control trials have investigated how minimally invasive approaches compare with open $\mathrm{CN}$.

\section{The cytokine period}

$\mathrm{CN}$ became standard of care for the treatment of mRCC after the publication of two landmark studies in 2001 . In SWOG-8949, patients with mRCC were randomly assigned to receive $\mathrm{CN}$ with the cytokine interferon alfa- $2 \mathrm{~b}$ (IFN $\alpha-2 b)$ or IFN $\alpha-2 b$ alone. Those undergoing the combined regimen of $\mathrm{CN}$ and cytokines demonstrated improved overall survival (OS) compared to cytokines alone (11.1 vs. 8.1 months, $\mathrm{P}=0.05$ ) (5). In EORTC-3047, Mickisch et al. randomized mRCC patients to receive $\mathrm{CN}$ with IFN $\alpha-2 \mathrm{~b}$ or IFN $\alpha-2 \mathrm{~b}$ alone. Patients in the $\mathrm{CN}$ plus cytokine arm also demonstrated increased OS (17 vs. 7 months, HR 0.54; 95\% CI: 0.31-0.94) as well as increased time to progression (15) (Table 1). Both studies demonstrated a perioperative mortality rate under $1 \%$ $(5,15)$. Pooled analysis of the SWOG and EORTC data showed that median survival was significantly greater for $\mathrm{CN}$ and IFN $\alpha-2 \mathrm{~b}$ vs. IFN $\alpha-2 \mathrm{~b}$ alone (13.6 vs. 7.8 months, HR 0.69; 95\% CI: $0.55-0.87, \mathrm{P}=0.002)$, irrespective of site of metastases and performance status (24). These results demonstrated that $\mathrm{CN}$ was a safe, life-prolonging treatment option for patients with mRCC who were treated with systemic cytokines $(5,15,24)$.

\section{The targeted therapy period}

As the understanding of tumor biology improved (25), TT was brought to bear on mRCC in the early 2000s. The number of agents in this class rapidly expanded and now includes drugs targeting vascular endothelial growth factor (VEGF) and its receptor (VEGFR) including [sunitinib, sorafenib, axitinib, pazopanib, cabozantinib, bevacizumab (with IFN $\alpha$ )], mTOR inhibitors (temsirolimus, everolimus), and combination therapy (lenvatinib plus everolimus). Multiple randomized trials demonstrated the superiority of these therapies over traditional cytokines (3). However, the majority of patients in these studies had already undergone $\mathrm{CN}$; therefore, it was not possible to determine the relative benefit of $\mathrm{CN}$ for patients being treated with TT.

There are several retrospective cohort studies that demonstrated a benefit to $\mathrm{CN}$ among patients treated with TT, although all suffered from significant selection bias 


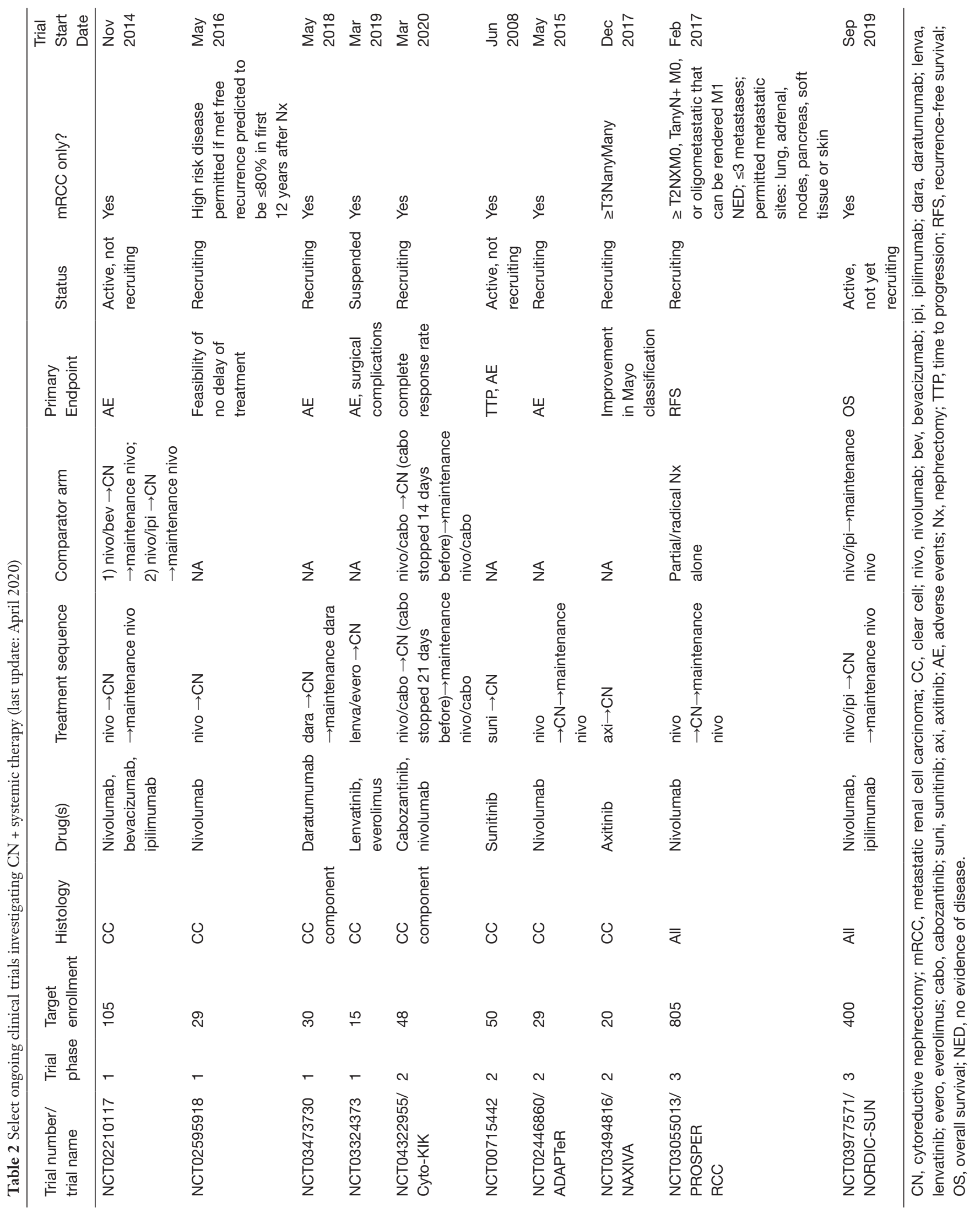


as patients who were offered $\mathrm{CN}$ were generally healthier than those not offered $\mathrm{CN}$. Choueiri et al. examined the role of $\mathrm{CN}$ in a retrospective review of 314 patients with mRCC treated VEGF-inhibitors. The 201 patients that had undergone $\mathrm{CN}$ were generally younger and had better performance status than those who did not undergo $\mathrm{CN}$. On multivariate analysis, the $\mathrm{CN}$ group had improved OS compared with the VEGF-inhibitor alone group (HR 0.68; 95\% CI: 0.46-0.99; P=0.04) (26). Moreover, favorable and intermediate risk patients demonstrated an OS advantage, which was not demonstrated in patients in the poor risk group (26,27). In a larger retrospective study surveying the National Cancer Database (NCDB), patients with mRCC who received TT were identified and grouped into those who did and did not receive $\mathrm{CN}$. Those who received $\mathrm{CN}$ were generally younger, had lower tumor stage, and no nodal disease. The $\mathrm{CN}$ group had increased OS compared with the non-surgical group (17.1 vs. 7.7 months; $\mathrm{P}<0.001$ ) and lower risk of mortality by any cause (HR $0.45 ; 95 \%$ CI: $0.40-0.50 ; \mathrm{P}<0.001)(28)$. While these and other studies support the use of $\mathrm{CN}$ in the setting of TT, the survival advantage from $\mathrm{CN}$ was lost in patients with poor risk disease $(3,26,28)$. These findings highlighted the need for randomized studies of $\mathrm{CN}$ with use of TT $(26,27)$.

\section{Patient selection for $\mathrm{CN}$}

Disease risk stratification for patients with mRCC is critical for assigning prognosis, systemic therapy, and selection for CN. The Memorial Sloan Kettering Cancer Center (MSKCC)/Motzer and International Metastatic Database Consortium (IMDC)/Heng criteria are two models that use clinical characteristics to categorize mRCC patients into favorable, intermediate, and poor risk groups which correlate with OS (29-31). Developed for use in the cytokine era, the MSKCC score combines serum hemoglobin, calcium, and $\mathrm{LDH}$, time from diagnosis to systemic therapy, and performance status (31). Similarly, the IMDC score takes into account serum hemoglobin and calcium, performance status, and time to receipt of systemic therapy but adds serum platelets and neutrophils (30). The IMDC model was specifically developed and validated for prognostication in patients with mRCC treated with TT (bevacizumab plus IFNa, sorafenib, and sunitinib) $(30,32)$. Additional serum laboratory tests such as C-reactive protein, albumin, and neutrophil-lymphocyte ration (NLR) may predict OS as well. It is important to note that presurgical prognostic grouping can change postoperatively, as some lab values may normalize after CN (4).

Retrospective studies have identified additional independent predictive factors to help select patients for $\mathrm{CN}$, although many of these likely reflect unmeasured surgeon selection criteria as the factors generally reflect better patient health (4). Well-established prognostic factors for patients who will not benefit from $\mathrm{CN}$ include older age, poor performance status, high burden of metastatic disease (particularly metastases to bone, brain, or liver), primary tumor invasion of adjacent organs, and sarcomatoid features $(4,7,33,34)$. Most recently, McIntosh et al. analyzed 608 mRCC patients who underwent $\mathrm{CN}$ at a single institution and identified five novel preoperative risk factors correlating with OS which included clinical T4 disease, retroperitoneal and supradiaphragmatic lymphadenopathy, hypoalbuminemia, and elevated lactate dehydrogenase as well as four previously reported risk factors such as systemic symptoms at diagnosis, bone metastasis, anemia, and neutrophil to lymphocyte ratio $\geq 4$ (35). The group detected a significant difference in median OS between those who had one, two, and three of the reported risk factors [58.9 months (95\% CI: 44.3-66.6 months) vs. 30.6 months (95\% CI: 27.0-35.0 months) vs. 19.3 months (95\% CI: 13.92.6-2 months)], respectively $(\mathrm{P}<0.0001)(35)$. They found that patients with adverse pathological features and poor risk disease tended to have a higher number of risk factors. These findings further illustrate the importance of using a risk-adapted approach in patient selection for $\mathrm{CN}(34,35)$.

Patient symptoms are another important aspect of selection for CN. $66 \%$ of patients with mRCC display symptoms with the most common being gross hematuria or flank pain from local tumor invasion, pain from bone metastasis, and dyspnea from lung metastasis (36). To assess the tradeoff between morbidity of surgery and improvement in symptoms, Larcher et al. reported on 317 patients with symptomatic mRCC treated with CN between 1988 and 2019. They found that after $\mathrm{CN}, 43 \%$ and $71 \%$ reported complete resolution or improvement in any symptoms related to their metastatic disease, respectively. With respect to local signs and symptoms, $91 \%$ and $95 \%$ demonstrated resolution and improvement, respectively. While the overall and major (Clavien Dindo $\geq 3$ ) complication rates were $37 \%$ and $10 \%$, respectively, the authors concluded that patients undergoing $\mathrm{CN}$ largely had a beneficial or mixed (symptom improvement/resolution with complication or no improvement/resolution without complication) response. IMDC risk group was not predictive of complication risk (36). 
In 2018, the CARMENA trial (NCT00930033) provided the first level one evidence about the role of $\mathrm{CN}$ in the setting of TT. In this non-inferiority study, 450 patients with MSKCC intermediate and poor risk clear cell mRCC were randomized to receive either $\mathrm{CN}$ followed by sunitinib or sunitinib alone. In the sunitinib only group, $17 \%$ ultimately required a $\mathrm{CN}$, whereas in the $\mathrm{CN}$-sunitinib group nearly $18 \%$ never received systemic therapy. After a median follow-up of 50.9 months, OS with sunitinib alone was non-inferior to $\mathrm{CN}$ followed by sunitinib [18.4 vs. 13.9 months (HR 0.89; 95\% CI: 0.71-1.10)] (16) (Table 1). These results corroborate prior reports that poor risk patients do not typically achieve survival benefit from $\mathrm{CN}$ (4), although for the first time, the use of $\mathrm{CN}$ in intermediate risk patients was less certain.

While this study introduced a potential paradigm shift in the use of $\mathrm{CN}$, the conclusions must be placed into context. Over $40 \%$ of patients in each arm had poor risk features, and OS was worse than contemporary studies (37), calling into question the external validity of the results. Also, the slow accrual of this study begets the possibility of recruitment bias, as healthier patients with fewer metastases may have chosen not to enroll (38). Arora et al. demonstrated that the CARMENA population might not necessarily be reflective of a general $\mathrm{mRCC}$ population by comparing CARMENA patients with NCDB patients who underwent $\mathrm{CN}$ and applying CARMENA inclusion criteria to the NCDB cohort. They found that the CARMENA cohort had lower OS, as well as more sites of metastasis and higher rates of metastases to the lymph node, lung, and bone compared to the NCDB cohort $(20,38)$. Ultimately, providers should use caution when applying the findings from CARMENA to their everyday practice and understand that the importance of judicious patient selection cannot be overstated. Continued efforts should be directed at the development of improved predictive models that consider both patient symptoms and risk factors, identifying reliable biomarkers $(39,40)$, and conducting larger randomized studies with higher proportions of good and intermediate risk patient populations undergoing $\mathrm{CN}$.

\section{Sequence of CN and TT}

In those who are deemed candidates for $\mathrm{CN}$, significant controversy exists over the sequence of performing surgery and initiating TT. Some argue for initial $\mathrm{CN}$, as decreasing tumor burden limits the number of tumor clones that can develop and spread. Alternatively, initial $\mathrm{CN}$ may delay the receipt of systemic therapy, permitting unrestricted metastatic growth (20).

To address the sequence of initiating $\mathrm{CN}$ and TT, a retrospective cohort study using the Surveillance, Epidemiology, and End Results (SEER) registry from 2006 to 2011 compared $190 \mathrm{mRCC}$ patients treated with initial $\mathrm{CN}$ and subsequent TT to 347 patients receiving initial TT, 28 of whom underwent delayed $\mathrm{CN}$ (41). When adjusted for clinical and pathologic factors, those treated with initial $\mathrm{CN}$ had a 5.8-month OS improvement compared to patients receiving TT (HR 0.50; 95\% CI: 0.38-0.65) (41).

In another retrospective study, Bhindi et al. identified patients who underwent either initial $\mathrm{CN}(\mathrm{n}=6,731)$ or TT $(n=8,337)$ in the NCDB from 2006-2013 (42). Nearly half of those receiving initial $\mathrm{CN}$ went on to receive TT, while only $4.9 \%$ of those receiving initial TT underwent $\mathrm{CN}$ within 6 months. OS was 16.5 vs. 9.2 months for patients undergoing upfront $\mathrm{CN} v s$. initial targeted therapy, respectively, (HR 0.61; 95\% CI: 0.59-0.64; $\mathrm{P}<0.001$ ). The authors concluded that upfront $\mathrm{CN}$ held a survival advantage over initial TT, as more patients had the opportunity to receive multimodal therapy (42).

In contrast to these results, a recent retrospective study of the IMDC database compared patients with mRCC who underwent $\mathrm{CN}$ followed by sunitinib $(\mathrm{n}=805)$, sunitinib followed by deferred $\mathrm{CN}(\mathrm{n}=85)$, or sunitinib alone $(\mathrm{n}=805)$. The authors observed that deferred $\mathrm{CN}$ patients had an OS advantage (HR 0.45; 95\% CI: 0.33-0.60; $\mathrm{P}<0.001$ ) over the other groups. When comparing all patients who underwent $\mathrm{CN}$, the OS advantage for deferred over upfront $\mathrm{CN}$ remained (HR 0.52; 95\% CI: 0.39-0.70; $\mathrm{P}<0.001$ ). Notably, only a fraction of patients included in the analysis were classified as poor risk (43). The mixed results from these studies highlight the limitations of retrospective cohort studies in guiding surgical selection. Patients who initially started on TT may have had poorer prognostic features prohibiting their surgical candidacy, underscored by the fact that only a minority underwent $\mathrm{CN}$ after TT in both studies favoring upfront CN $(41,42)$. However, carefully selected patients who are healthy enough to undergo a $\mathrm{CN}$ may live significantly longer if TT is employed first (43).

SURTIME (NCT01099423), the first prospective data regarding the optimal sequence for TT and $\mathrm{CN}$, was published in 2019. In this phase 3 trial, patients were randomized to receive upfront $\mathrm{CN}$ followed by sunitinib ( $\mathrm{n}=50)$ or sunitinib followed by $\mathrm{CN}$ if patients had not progressed on therapy $(n=49)$. Due to poor accrual, enrollment ended early, although intention-to-treat analysis 
revealed a median OS of $32.4 v s .15 .0$ months, for the delayed and initial $\mathrm{CN}$ arms, respectively (HR 0.57; $95 \%$ CI: 0.34-0.95; $\mathrm{P}=.03$ ) (17) (Table 1). While underpowered, SURTIME suggested that receiving systemic TT prior to $\mathrm{CN}$ was beneficial, as it may aid in controlling metastatic disease. Furthermore, administration of preoperative TT may guide in patient selection for $\mathrm{CN}$, as patients who progress on TT may not benefit from CN (7). Future prospective studies with larger cohorts are needed to further clarify the proper sequence of TT and CN.

\section{CN and active surveillance}

In some patients with mRCC, the growth of metastases is indolent. Several groups have utilized active surveillance (AS), or treatment delay (TD) with TT, in highly select patients with low-volume mRCC to avoid inherent toxicity and costs associated with systemic treatment (1). In a prospective phase 2 trial, 48 patients with asymptomatic, TT-naïve mRCC underwent radiographic surveillance until TT was deemed necessary by their providers. Of this cohort, $98 \%$ had already undergone nephrectomy, but the rate of primary nephrectomy $v$ s. CN was not stated. Median time to progression was 9.4 months (95\% CI: 7.4-13.4), and median AS time was 14.9 months (95\% CI: 10.6-25.0 months). Prognostic factors associated with longer periods of AS included fewer sites of metastasis and fewer IMDC risk factors. Interestingly, patient anxiety, depression, and quality of life (QoL) scores did not change during the AS period (44).

In a subsequent retrospective review of the NCDB, Woldu et al. examined mRCC patients who underwent delayed treatment with TT after CN. Median TD was 2.1 months, and the majority of patients began TT within 4 months after CN. TD was not independently predictive of OS (1). More recently, Iacovelli et al. reported data from 16 Italian hospitals on 635 patients with mRCC who were deemed eligible for TT, but opted for AS. Of those who were metastatic at diagnosis, $68.7 \%$ had undergone $\mathrm{CN}$. Median OS was 27.7 months (95\% CI: 24.8-30.5), and median progression free survival (PFS) was 11.1 months (95\% CI: 9.9-12.3) among all patients. TD was 8 weeks for the $\mathrm{CN}$ patients compared to 5.3 weeks for those who underwent nephrectomy for initially localized disease $(\mathrm{P}=0.001)$. However, TD after $\mathrm{CN}$ did not affect OS (45). Taken together, these studies provide evidence that AS may be a safe initial treatment strategy in some carefully selected mRCC patients and does not necessitate and tradeoff between survival and QoL $(1,44,45)$.

\section{The immune checkpoint inhibitor period and future directions}

Systemic IO agents for $\mathrm{mRCC}$ are being rapidly introduced, and immune checkpoint inhibitors (ICIs) (46) targeting programed cell death 1 protein (PD-1) (nivolumab, pembrolizumab), programed death-ligand 1 (PD-L1) (avelumab), and CTLA-4 (ipilimumab) have been shown to be superior as first-line therapy for many patients with mRCC (19). In the phase 3 trial Checkmate-214 (NCT02231749), patients with untreated advanced RCC were risk-stratified using IMDC criteria and randomized to receive either sunitinib $(\mathrm{n}=546)$ or nivolumab/ipilimumab $(\mathrm{n}=550) .80 \%$ of the nivolumab/ipilimumab and $76 \%$ of the sunitinib group had a previous nephrectomy. Among patients with intermediate and poor risk RCC, the 18-month OS for nivolumab/ipilimumab vs. sunitinib was $75 \%$ vs. $60 \%$, respectively (HR $0.63 ; 99.8 \%$ CI: $0.44-0.89 ; \mathrm{P}<0.001)$. The nivolumab/ipilimumab group had a higher objective and complete response rate than the sunitinib group (37). The open-label phase 3 trial KEYNOTE-426 (NCT02853331) similarly randomized patients with treatment naïve advanced RCC to receive either pembrolizumab with axitinib $(\mathrm{n}=432)$ or sunitinib $(n=429)$. Across all IMDC risk groups, patients in the pembrolizumab/axitinib arm exhibited improved OS (HR 0.53; 95\% CI: $0.38-0.74 ; \mathrm{P}<0.0001)$ than the sunitinib group (37). In JAVELIN Renal 101 (NCT02684006), advanced RCC patients were randomized to receive avelumab plus axitinib $(n=442)$ or sunitinib alone $(n=444)$. The median PFS in the overall population was 13.8 vs. 8.4 months (HR 0.69; 95\% CI: 0.56-0.84; $\mathrm{P}<0.001$ ) for the avelumab/axitinib $v s$. sunitinib groups, respectively, with remarkably similar results for the subset of patients with PD-L1 positive tumors. Notably, over $99 \%$ of patients in both groups experienced adverse events (47). The promising results from these trials led to the approval of these combination therapies for advanced RCC, although toxicities must be taken into account when selecting patients for treatment.

As systemic therapies for mRCC rapidly evolve, the role of $\mathrm{CN}$ requires ongoing reevaluation. In a retrospective study using the NCDB, Singla et al. analyzed 391 patients who received either $\mathrm{CN}$ and IO $(n=221)$ or IO alone $(\mathrm{n}=170)$. They observed that patients who underwent $\mathrm{CN}$ and IO had lower pT stage, tumor grade and size, and 


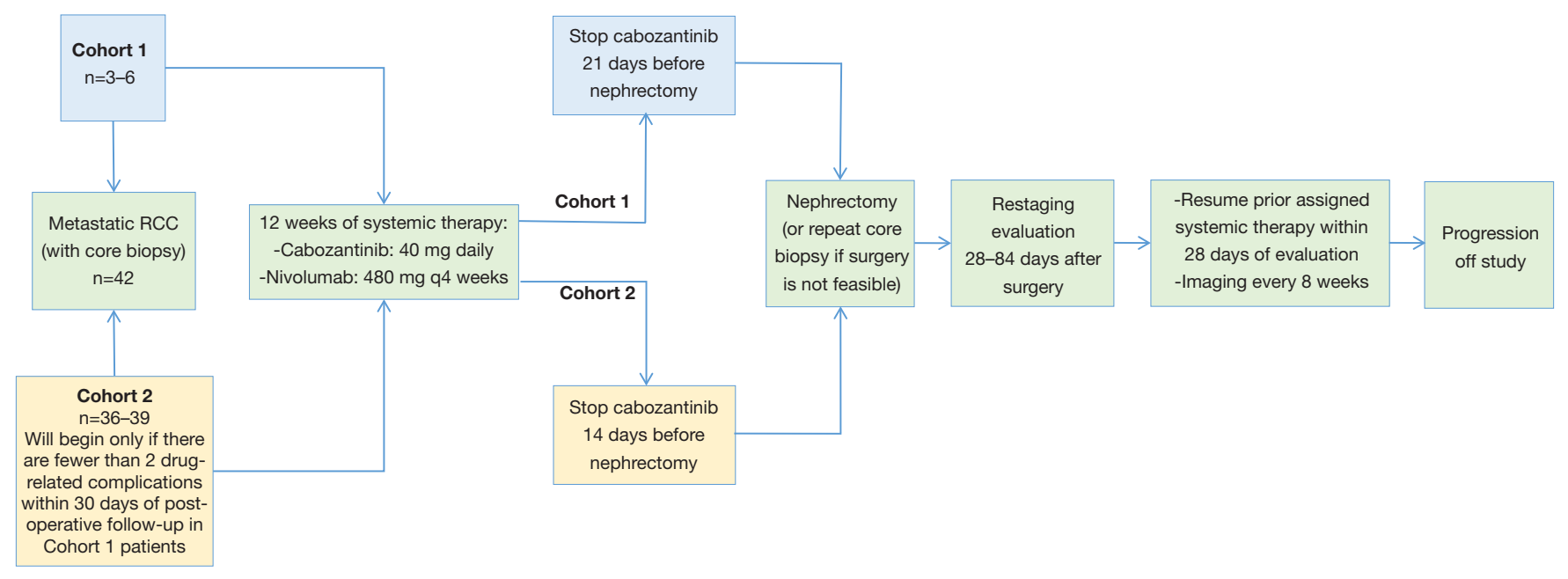

Figure 1 Schematic of Cyto-KIK Trial (NCT04322).

rates of lymphovascular invasion compared to the $\mathrm{IO}$ alone group. After a median follow-up of 14.7 months, the $\mathrm{CN}$ and IO group demonstrated superior OS compared to those receiving IO alone (not reached vs. 11.6 months; HR 0.23; $\mathrm{P}<0.001)$. Again, patients selected for $\mathrm{CN}$ may have had more favorable patient and tumor characteristics that contributed to their superior outcomes (48). Therefore, clinical trials are required to investigate the role of $\mathrm{CN}$ in the setting of IO therapy, as well as the sequencing of $\mathrm{CN}$ and IO systemic therapy (Table 2).

Compelling preclinical data in other solid tumors suggests a beneficial role for neoadjuvant IO prior to cytoreductive surgery. In a study of glioblastoma patients randomized to neoadjuvant or adjuvant pembrolizumab, the neoadjuvant group had significantly improved OS (13.7 vs. 7.5 months; HR 0.39; 95\% CI: 0.17-0.94; $\mathrm{P}=0.04$ ) (49). In patients with metastatic melanoma, the OpACIN study demonstrated that those treated with ipilimumab/nivolumab prior to surgery had an improved pathological response rate and relapse free survival $(80 \%$ vs. $60 \%)$ than those who received adjuvant IO therapy $(50,51)$. While the mechanisms underlying the clinical benefits of neoadjuvant IO are not fully understood, it is postulated that neoadjuvant IOs cause dying tumor cells to release tumor-specific antigens, which may prime the immune system by enhancing $\mathrm{T}$ cell proliferation and amplifying the anti-tumor response $(49,51,52)$.

The combination of studies showing a benefit for neoadjuvant IO in others solid tumors and recent literature demonstrating improved OS with deferred $\mathrm{CN}$ supports the need for a clinical trial examining neoadjuvant IO and deferred CN. The Cyto-KIK trial (NCT04322955) is a phase 2 multi-center trial investigating the impact of neoadjuvant nivolumab and cabozantinib (a VEGFR inhibitor) followed by $\mathrm{CN}$ and continued systemic therapy. The primary clinical endpoint is complete response rate with secondary endpoints including OS, PFS, primary tumor size reduction, toxicity, and surgical complications. Moreover, the investigators intend to use pre-treatment RCC tissue samples to identify biomarkers indicating response to TT and IO. The trial opened in 2020 with a target enrollment of 48 patients (Figure 1) (53).

\section{Summary}

$\mathrm{CN}$ is a rapidly changing treatment option for patients with mRCC. Before offering $\mathrm{CN}$, clinicians should carefully select patients using pre-operative patient risk stratification tools; they should counsel patients on risk of surgery as well as benefits, including possible increased survival and symptomatic improvement. Moreover, some patients can be offered systemic therapies, but the optimal timing with surgery is still an active area of research.

This narrative review has inherent limitations as it is non-systematic. As with all non-systematic reviews, there is a possibility for bias, as the evidence provided has not been systematically evaluated. However, our intention is to provide a comprehensive overview on various aspects of $\mathrm{CN}$, place its evolution into historical context, and highlight up-and-coming research. Additionally, most of the studies cited were retrospective in nature, as there is still a paucity 
of level one evidence detailing the precise clinical role of $\mathrm{CN}$. Randomized clinical trials will be critical for further understanding how to best use $\mathrm{CN}$ in practice.

\section{Conclusions}

The role of $\mathrm{CN}$ has evolved as the landscape of systemic therapy for $\mathrm{mRCC}$ has changed. While receipt of immediate TT has become standard for many patients, CN remains an important treatment option in carefully selected patients with good or intermediate risk mRCC. Because many questions remain regarding the optimal systemic therapy regimen and timing of surgery, patients interested in $\mathrm{CN}$ should be encouraged to do so as part of a clinical trial.

\section{Acknowledgments}

Funding: This work was supported by a grant from the National Cancer Institute [P30CA072720].

\section{Footnote}

Reporting Checklist: The authors have completed the Narrative Review reporting checklist. Available at http:// dx.doi.org/10.21037/tcr-20-2343

Peer Review File: Available at http://dx.doi.org/10.21037/tcr20-2343

Conflicts of Interest: All authors have completed the ICMJE uniform disclosure form (available at http://dx.doi. org/10.21037/tcr-20-2343). Dr. EAS serves as an unpaid editorial board member of Translational Cancer Research from Jan 2019 to Dec 2020. Dr. EAS reports grants from Astellas/Medivation, outside the submitted work. Dr. MNS reports grants from Janssen Oncology, grants from Advaxis, grants from Harpoon, grants from Bristol-Meyers Squibb, grants from Genocea Biosciences, grants from Lilly, grants from Nektar, grants from Seattle Genetics, grants from Xencor, grants from Tmunity, grants from Exelixis, during the conduct of the study. Dr. CGD reports grants from AZ Medimmune, grants from Bayer, grants from BristolMeyers Squibb, grants and other from Compugen, grants from Ferring, grants from F-Star, grants from Genocea, grants from Janssen, grants and other from Kleo, grants from Merck, grants from Merck-Serono, grants from Pfizer, grants from Pierre Fabre, grants from Roche/Genentech, grants from Shattuck Labs, grants from Tizona, grants and other from Urogen, grants and other from Werewolf, other from Harpoon, outside the submitted work. The other authors have no conflicts of interest to declare.

Ethical Statement: The authors are accountable for all aspects of the work in ensuring that questions related to the accuracy or integrity of any part of the work are appropriately investigated and resolved.

Open Access Statement: This is an Open Access article distributed in accordance with the Creative Commons Attribution-NonCommercial-NoDerivs 4.0 International License (CC BY-NC-ND 4.0), which permits the noncommercial replication and distribution of the article with the strict proviso that no changes or edits are made and the original work is properly cited (including links to both the formal publication through the relevant DOI and the license). See: https://creativecommons.org/licenses/by-nc-nd/4.0/.

\section{References}

1. Woldu SL, Matulay JT, Clinton TN, et al. Incidence and Outcomes of Delayed Targeted Therapy After Cytoreductive Nephrectomy for Metastatic Renal-Cell Carcinoma: A Nationwide Cancer Registry Study. Clin Genitourin Cancer 2018;16:e1221-35.

2. Kidney Cancer: Statistics. American Society of Clinical Oncology (ASCO). 2019. Available online: https://www. cancer.net/cancer-types/kidney-cancer/statistics. Accessed December 6, 2019.

3. Soares A, Maia MC, Vidigal F, et al. Cytoreductive Nephrectomy for Metastatic Renal Cell Carcinoma: How to Apply New Evidence in Clinical Practice. Oncology 2020;98:1-9.

4. Shapiro DD, Abel EJ. Patient selection for cytoreductive nephrectomy in combination with targeted therapies or immune checkpoint inhibitors. Curr Opin Urol 2019;29:513-20.

5. Flanigan RC, Salmon SE, Blumenstein BA, et al. Nephrectomy followed by interferon alfa-2b compared with interferon alfa-2b alone for metastatic renal-cell cancer. N Engl J Med 2001;345:1655-9.

6. Marcus SG, Choyke PL, Reiter R, et al. Regression of metastatic renal cell carcinoma after cytoreductive nephrectomy. J Urol 1993;150:463-6.

7. Bhindi B, Abel EJ, Albiges L, et al. Systematic Review of the Role of Cytoreductive Nephrectomy in the 
Targeted Therapy Era and Beyond: An Individualized Approach to Metastatic Renal Cell Carcinoma. Eur Urol 2019;75:111-28.

8. Singh AK, Winslow TB, Kermany MH, et al. A Pilot Study of Stereotactic Body Radiation Therapy Combined with Cytoreductive Nephrectomy for Metastatic Renal Cell Carcinoma. Clin Cancer Res 2017;23:5055-65.

9. Laurell A, Lonnemark M, Brekkan E, et al. Intratumorally injected pro-inflammatory allogeneic dendritic cells as immune enhancers: a first-in-human study in unfavourable risk patients with metastatic renal cell carcinoma. J Immunother Cancer 2017;5:52.

10. Oudard S, Mejean A, Topart D, et al. 880P Biomarkers before and after nephrectomy of locally advanced or metastatic renal cell carcinoma (RCC) treated with everolimus: Neorad phase 2 trial (PREDICT consortium). Ann Oncol 2017;28:V310.

11. Lindskog M, Laurell A, Kjellman A, et al. A randomized phase II study with ilixadencel, a cell-based immune primer, plus sunitinib versus sunitinib alone in synchronous metastatic renal cell carcinoma. J Clin Oncol 2020;38:11.

12. de Bruijn R, Wimalasingham A, Szabados B, et al. Deferred Cytoreductive Nephrectomy Following Presurgical Vascular Endothelial Growth Factor Receptortargeted Therapy in Patients with Primary Metastatic Clear Cell Renal Cell Carcinoma: A Pooled Analysis of Prospective Trial Data. Eur Urol Oncol 2020;3:168-73.

13. Bex A, Blank C, Meinhardt W, et al. A phase II study of presurgical sunitinib in patients with metastatic clear-cell renal carcinoma and the primary tumor in situ. Urology 2011;78:832-7.

14. Powles T, Sarwar N, Stockdale A, et al. Safety and Efficacy of Pazopanib Therapy Prior to Planned Nephrectomy in Metastatic Clear Cell Renal Cancer. JAMA Oncol 2016;2:1303-9.

15. Mickisch GH, Garin A, van Poppel H, et al. Radical nephrectomy plus interferon-alfa-based immunotherapy compared with interferon alfa alone in metastatic renal-cell carcinoma: a randomised trial. Lancet 2001;358:966-70.

16. Méjean A, Ravaud A, Thezenas S, et al. Sunitinib Alone or after Nephrectomy in Metastatic Renal-Cell Carcinoma. N Engl J Med 2018;379:417-27.

17. Bex A, Mulders P, Jewett $M$, et al. Comparison of Immediate vs Deferred Cytoreductive Nephrectomy in Patients With Synchronous Metastatic Renal Cell Carcinoma Receiving Sunitinib: The SURTIME Randomized Clinical Trial. JAMA Oncol 2019;5:164-70.
18. Mauge L, Mejean A, Fournier L, et al. Sunitinib Prior to Planned Nephrectomy in Metastatic Renal Cell Carcinoma: Angiogenesis Biomarkers Predict Clinical Outcome in the Prospective Phase II PREINSUT Trial. Clin Cancer Res 2018;24:5534-42.

19. Kidney Cancer (Version 2.2020). National Comprehensive Cancer Network. 2019. Available online: https://www. nccn.org/professionals/physician_gls/pdf/kidney.pdf. Accessed December 12, 2019.

20. Bex A, Albiges L, Ljungberg B, et al. Updated European Association of Urology Guidelines for Cytoreductive Nephrectomy in Patients with Synchronous Metastatic Clear-cell Renal Cell Carcinoma. Eur Urol 2018;74:805-9.

21. Gershman B, Moreira DM, Boorjian SA, et al. Comprehensive Characterization of the Perioperative Morbidity of Cytoreductive Nephrectomy. Eur Urol 2016;69:84-91.

22. Roussel E, Campi R, Larcher A, et al. Rates and Predictors of Perioperative Complications in Cytoreductive Nephrectomy: Analysis of the Registry for Metastatic Renal Cell Carcinoma. Eur Urol Oncol 2020;3:523-9.

23. Nunez Bragayrac L, Hoffmeyer J, Abbotoy D, et al. Minimally invasive cytoreductive nephrectomy: a multiinstitutional experience. World J Urol 2016;34:1651-6.

24. Flanigan RC, Mickisch G, Sylvester R, et al. Cytoreductive nephrectomy in patients with metastatic renal cancer: a combined analysis. J Urol 2004;171:1071-6.

25. William G. Kaelin Jr. Facts. The Nobel Prize. 2020. https://www.nobelprize.org/prizes/medicine/2019/kaelin/ facts/. 2020

26. Choueiri TK, Xie W, Kollmannsberger C, et al. The impact of cytoreductive nephrectomy on survival of patients with metastatic renal cell carcinoma receiving vascular endothelial growth factor targeted therapy. J Urol 2011;185:60-6.

27. Singer EA, Srinivasan R, Bratslavsky G. Editorial comment. J Urol 2011;185:66.

28. Hanna N, Sun M, Meyer CP, et al. Survival Analyses of Patients With Metastatic Renal Cancer Treated With Targeted Therapy With or Without Cytoreductive Nephrectomy: A National Cancer Data Base Study. J Clin Oncol 2016;34:3267-75.

29. Heng DY, Xie W, Regan MM, et al. External validation and comparison with other models of the International Metastatic Renal-Cell Carcinoma Database Consortium prognostic model: a population-based study. Lancet Oncol 2013;14:141-8.

30. Heng DY, Xie W, Regan MM, et al. Prognostic factors 
for overall survival in patients with metastatic renal cell carcinoma treated with vascular endothelial growth factortargeted agents: results from a large, multicenter study. J Clin Oncol 2009;27:5794-9.

31. Motzer RJ, Mazumdar M, Bacik J, et al. Survival and prognostic stratification of 670 patients with advanced renal cell carcinoma. J Clin Oncol 1999;17:2530-40.

32. Kwon WA, Cho IC, Yu A, et al. Validation of the MSKCC and Heng risk criteria models for predicting survival in patients with metastatic renal cell carcinoma treated with sunitinib. Ann Surg Oncol 2013;20:4397-404.

33. Culp SH, Tannir NM, Abel EJ, et al. Can we better select patients with metastatic renal cell carcinoma for cytoreductive nephrectomy? Cancer 2010;116:3378-88.

34. Psutka SP. Personalizing preoperative risk stratification and refining patient selection for cytoreductive nephrectomy in metastatic renal cell carcinoma. Cancer 2020;126:3912-5.

35. McIntosh AG, Umbreit EC, Holland LC, et al. Optimizing patient selection for cytoreductive nephrectomy based on outcomes in the contemporary era of systemic therapy. Cancer 2020;126:3950-60.

36. Larcher A, Fallara G, Rosiello G, et al. Cytoreductive Nephrectomy in Metastatic Patients with Signs or Symptoms: Implications for Renal Cell Carcinoma Guidelines. Eur Urol 2020;78:321-6.

37. Motzer RJ, Tannir NM, McDermott DF, et al. Nivolumab plus Ipilimumab versus Sunitinib in Advanced Renal-Cell Carcinoma. N Engl J Med 2018;378:1277-90.

38. Arora S, Sood A, Dalela D, et al. Cytoreductive Nephrectomy: Assessing the Generalizability of the CARMENA Trial to Real-world National Cancer Data Base Cases. Eur Urol 2019;75:352-3.

39. Farber NJ, Kim CJ, Modi PK, et al. Renal cell carcinoma: the search for a reliable biomarker. Transl Cancer Res 2017;6:620-32.

40. Modi PK, Farber NJ, Singer EA. Precision Oncology: Identifying Predictive Biomarkers for the Treatment of Metastatic Renal Cell Carcinoma. Transl Cancer Res 2016;5:S76-S80.

41. Macleod LC, Odisho AY, Tykodi SS, et al. Comparative Effectiveness of Initial Surgery vs Initial Systemic Therapy for Metastatic Kidney Cancer in the Targeted Therapy Era: Analysis of a Population-based Cohort. Urology 2018;113:146-52.

42. Bhindi B, Habermann EB, Mason RJ, et al. Comparative Survival following Initial Cytoreductive Nephrectomy versus Initial Targeted Therapy for Metastatic Renal Cell Carcinoma. J Urol 2018;200:528-34.
43. Bhindi B, Graham J, Wells JC, et al. Deferred Cytoreductive Nephrectomy in Patients with Newly Diagnosed Metastatic Renal Cell Carcinoma. Eur Urol 2020;78:615-23.

44. Rini BI, Dorff TB, Elson P, et al. Active surveillance in metastatic renal-cell carcinoma: a prospective, phase 2 trial. Lancet Oncol 2016;17:1317-24.

45. Iacovelli R, Galli L, De Giorgi U, et al. The effect of a treatment delay on outcome in metastatic renal cell carcinoma. Urol Oncol 2019;37:529.e1-e7.

46. James P. Allison Facts. The Nobel Prize. 2020. Available online: https://www.nobelprize.org/prizes/medicine/2018/ allison/facts/

47. Motzer RJ, Penkov K, Haanen J, et al. Avelumab plus Axitinib versus Sunitinib for Advanced Renal-Cell Carcinoma. N Engl J Med 2019;380:1103-15.

48. Singla N, Hutchinson RC, Ghandour RA, et al. Improved survival after cytoreductive nephrectomy for metastatic renal cell carcinoma in the contemporary immunotherapy era: An analysis of the National Cancer Database. Urol Oncol 2020;38:604.e9-604.e17.

49. Cloughesy TF, Mochizuki AY, Orpilla JR, et al. Neoadjuvant anti-PD-1 immunotherapy promotes a survival benefit with intratumoral and systemic immune responses in recurrent glioblastoma. Nat Med 2019;25:477-86.

50. Blank CU, Rozeman EA, Fanchi LF, et al. Neoadjuvant versus adjuvant ipilimumab plus nivolumab in macroscopic stage III melanoma. Nat Med 2018;24:1655-61.

51. Killock D. New window of opportunity with ICIs in melanoma. Nat Rev Clin Oncol 2018;15:723.

52. Liu J, Blake SJ, Yong MC, et al. Improved Efficacy of Neoadjuvant Compared to Adjuvant Immunotherapy to Eradicate Metastatic Disease. Cancer Discov 2016;6:1382-99.

53. CYTO Reductive Surgery in Kidney Cancer Plus Immunotherapy and Targeted Kinase Inhibition (CytoKIK): U.S. National Library of Medicine; 2020. Available online: https://clinicaltrials.gov/ct2/show/ NCT04322955.

Cite this article as: Tabakin AL, Stein MN, Anderson $\mathrm{CB}$, Drake CG, Singer EA. Cytoreductive nephrectomy for metastatic renal cell carcinoma, the ultimate urologic 'Choosing Wisely' campaign: a narrative review. Transl Cancer Res 2020;9(11):7337-7349. doi: 10.21037/tcr-20-2343 